\title{
Competencias más importantes para la disciplina administrativa en Colombia
}

\author{
Most important competences for the management discipline in Colombia \\ Jaime Castrillón, Leonor Cabeza y Jahir Lombana* \\ Universidad del Norte, Escuela de Negocios, Colombia
}

Recibido el 31 de octubre de 2014; aceptado el 18 de febrero de 2015

Disponible en Internet el 22 de agosto de 2015

\begin{abstract}
Resumen
A partir de los resultados encontrados del Proyecto Tuning Latin-America, que se replicó en Colombia, esta investigación identifica de las 47 competencias del proyecto, las 5 más importantes según la percepción de los académicos y de los empleadores, las cuales deben ser tenidas en cuenta para garantizar buenos resultados de los profesionales en administración. Las competencias también se han clasificado de acuerdo con los fundamentos filosóficos de Bédard en ontológicas, axiológicas, epistemológicas, praxiológicas. Este estudio usa una orientación descriptiva cuando se construye el ranking de las competencias y aplica una inferencia estadística para determinar el nivel de importancia de ellas. Los resultados muestran una percepción diferente para académicos y empleadores. Sin embargo, empleadores y académicos piensan que los administradores están orientados a los elementos epistemológicos y praxiológicos, tanto en las competencias genéricas como específicas. Se puede concluir que es necesario hacer un esfuerzo para cerrar la brecha entre los conocimientos y los aspectos prácticos frente a los conceptos éticos, morales y los principios ontológicos mediante una buena educación impartida en las instituciones de educación superior.

Derechos Reservados ( 2015 Universidad Nacional Autónoma de México, Facultad de Contaduría y Administración. Este es un artículo de acceso abierto distribuido bajo los términos de la Licencia Creative Commons CC BY-NC-ND 4.0.
\end{abstract}

Palabras clave: Competencias; Educación superior; Administración; Fundamentos filosóficos; Proyecto Tuning

\footnotetext{
* Autor para correspondencia.

Correo electrónico: lombanaj@uninorte.edu.co (J. Lombana).

La revisión por pares es responsabilidad de la Universidad Nacional Autónoma de México.
} 


\begin{abstract}
Based on results of the Tuning Latin-America Project, replicated in Colombia, this research identifies the 5 most important competencies according to the mean of perception from academicians and employers to be taken into account for the performance of administration professionals. Competencies are classified according to the philosophical foundations taking into account Bédard's approach of: ontological, axiological, epistemological and praxeological. This study uses a descriptive orientation on the construction of a ranking and applies statistical inference to determine the level of importance of competencies. Results show different perceptions between academicians and employers. Employers and academicians think that managers are oriented to the epistemology and praxeology, both for generic and specific competencies. It can be concluded that it is necessary to close the gap between the knowledge and praxis versus values and principles through education in higher education institutions.

All Rights Reserved () 2015 Universidad Nacional Autónoma de México, Facultad de Contaduría y Administración. This is an open access item distributed under the Creative Commons CC License BY-NC-ND 4.0.
\end{abstract}

Keywords: Competences; Higher education; Management; Philosophical foundations; Tuning Project

\title{
Introducción
}

Esta investigación está soportada teóricamente en el proyecto Tuning Educational Structures in Europe (2000-2010) (Gonzalez y Wagenaar, 2006) y el análisis de fundamentos filosóficos de Bédard (2003). Tuning comenzó en Europa como respuesta a los acuerdos firmados en materia de educación en el proceso de Bolonia. El proyecto fue replicado en América Latina desde el punto de vista metodológico y más tarde en Colombia (ASCOLFA-GRIICA, 2010). En la orientación colombiana se determinó el grado de importancia y desarrollo de las competencias genéricas y específicas para profesionales de la administración, a través de una encuesta de percepción sobre competencias genéricas y específicas. Por otra parte, el trabajo realizado por Bédard asume que los gerentes deben tener 4 fundamentos filosóficos, a saber: epistemológico (conocimiento), praxeológico (práctica), axiológico (valores) y ontológico (individuo y su identidad como ser humano). Según Bédard (2003), estas 4 dimensiones están estrechamente coordinadas y su afinidad es inobjetable.

El objetivo de esta investigación es identificar, probar, jerarquizar y clasificar las 5 competencias genéricas y las 5 específicas más importantes y desarrolladas que se deben tener en la disciplina administrativa en Colombia, según la percepción de empleadores y académicos.

Esta investigación utiliza la base de datos del proyecto Tuning Colombia con el fin de identificar la importancia y desarrollo de las mejores competencias según el punto de vista de empleadores y académicos, y las clasifica bajo el enfoque de Bédard según los fundamentos filosóficos. Este trabajo se inicia con un marco conceptual que propone un debate sobre los conceptos y la clasificación de las competencias, haciendo especial énfasis en el enfoque Bédard. Seguidamente se expone la metodología y a continuación, se desarrollan el análisis y resultados. En esta etapa se mide la importancia de las competencias genéricas y específicas del proyecto Tuning para luego ordenarlas en forma descendente. La inferencia estadística se utiliza para determinar las 5 más importantes, se prueba la hipótesis nula: el promedio de importancia de la competencia está por encima de 3, es decir, es importante o muy importante. Después de este ranking, las competencias se evalúan en función de su desarrollo. Por último, las competencias se asocian con el enfoque Bédard y se presentan algunas conclusiones. 


\section{Aspectos teóricos}

Para una revisión del concepto de competencia se toman varios autores tales como: Mulder (2007); Tejada (1999); Hoffmann (1999); Ferrández (1997); Le Boterf (1994), también se debate la clasificación en el escenario académico por ejemplo con Beneitone, Esqueti, González, Marty, Siufi y Wagenaar (2007) y Bunk (1994). De esta revisión se acoge la definición de competencia de Tuning Europa (Gonzalez y Wagenaar, 2006) como la combinación dinámica de conocimiento, comprensión, capacidades y habilidades.

El concepto de competencia se utiliza de muchas maneras, dependiendo del contexto, cultura, historia, objetivos o intereses; esto crea dificultades o genera discusiones. El concepto de competencia otorga un significado de unidad e implica que los elementos del conocimiento tienen sentido solo en función del conjunto, por esto la formación de la universidad debe partir de una formación profesional que, además de promover el desarrollo de ciertos atributos (habilidades, conocimientos, aptitudes, actitudes y valores), considere la ocurrencia de varias tareas (acciones intencionales) que suceden simultáneamente dentro del contexto (Cabrera y González, 2006).

«El Banco Mundial confirma que la formación y el desarrollo de competencias es un papel que las instituciones de educación superior deben cumplir con el plan de estudios, entendido como un conjunto estructurado de conocimientos para intencionalmente articular las experiencias de aprendizaje con el fin de producir cambios en las formas de sentir, pensar y actuar en los que pasan a través de él». (Cabrera, Guzmán, Suárez y Rincón, 2009. p.8). Además, Bunk (1994) dice que la educación en competencias es necesaria para el proceso de transmisión de conocimientos que comienza el primer día de entrenamiento y continúa en el lugar de trabajo. Argumenta que el estudio y la transmisión aislada no alcanzan el objetivo; el enfoque de Bédard es una de las formas de llegar a estos objetivos, según lo propuesto por el Banco Mundial y Bunk, de manera integradora y global. A esto se suman las opiniones de los aspectos axiológicos y ontológicos como partes de un proceso integrador.

El concepto de competencia tiene una larga historia. Mulder (2007) dice que el Código de Hammurabi (1792-1750 a. de C) lo incluye por primera vez, pero parece que el concepto aparece en las culturas occidentales sobre el siglo XV con la raíz latina competere que significa competir (Quillet, 1976 p.633). En este sentido, el concepto tiene que ver con la lucha como la confrontación para convertirse en el mejor (Cabeza, Castrillón y Lombana, 2012).

Aunque la aplicación académica del concepto puede remontarse a Max Weber (1921) con su trabajo sobre la burocracia, como una serie de funciones delimitadas, con poderes de realización y con la fijación de medios incluso coercitivos para su aplicación, su acepción puede remitir más al término autoridad (Behörde) que al de competencia (Kompetenz). Es más, con el trabajo de McClelland (1973) se recogen nuevamente los temas de aptitud e idoneidad, particularmente en el campo educativo.

Gonczi (1997) dice que el enfoque integrado de la competencia se produce por la combinación de atributos, tareas y contextos. Este reúne las críticas de los diferentes enfoques de competencia que se encuentran en la literatura, lo que permite la incorporación de la ética y los valores como elementos del desempeño competente, la necesidad de la práctica reflexiva, la importancia del contexto cultural y el hecho de que podría haber más de una manera de trabajar de forma competente. Según Beneitone et al. (2007, p. 37) «representa una combinación dinámica de conocimientos, comprensión, habilidades y capacidades. El fomento de las competencias es el objetivo de los programas educativos. Las competencias se forman en varias unidades del curso y son evaluadas en diferentes etapas». 
Hoffmann (1999) y Ruiz (2005) clasifican las competencias en 3 grupos: a) Conductual: hace referencia a los resultados. Ducci (1997); Mertens (1997) y Gallart (1998) plantean que se espera que las normas guíen el desarrollo del comportamiento. Este grupo apoya el concepto de competencia laboral, pone la definición en contextos de trabajo, en situaciones de incertidumbre y la forma de armonizar los sistemas de producción con la educación. En el área administrativa autores como Boyatzis (1982) y Mole, Dawson, Winstanley y Sherval (1993) están de acuerdo en este enfoque y consideran la educación como el camino para dar forma a las actitudes y atributos. b) Constructivista; define las competencias del ser humano, con sus acciones, el rendimiento y las capacidades mentales. Según Vygotsky (1979), la competencia debe ser la capacidad del gestor para adaptar sus decisiones, hábilmente y con antelación, frente a la evolución del contexto. Este enfoque construye la competencia basada en el contexto y las tareas demandadas (Bunk, 1994; Mintzberg, 1973; Victorino y Medina, 2008; UNESCO, 2011). c) Funcional: este enfoque da prioridad al resultado, no importa el proceso. Las habilidades, conocimientos y las respuestas dadas a un problema definen las competencias. Los resultados son el único indicador para definir la competencia. (Gilbert, 1978; Koshansky, 1998 y Malpica, 2000).

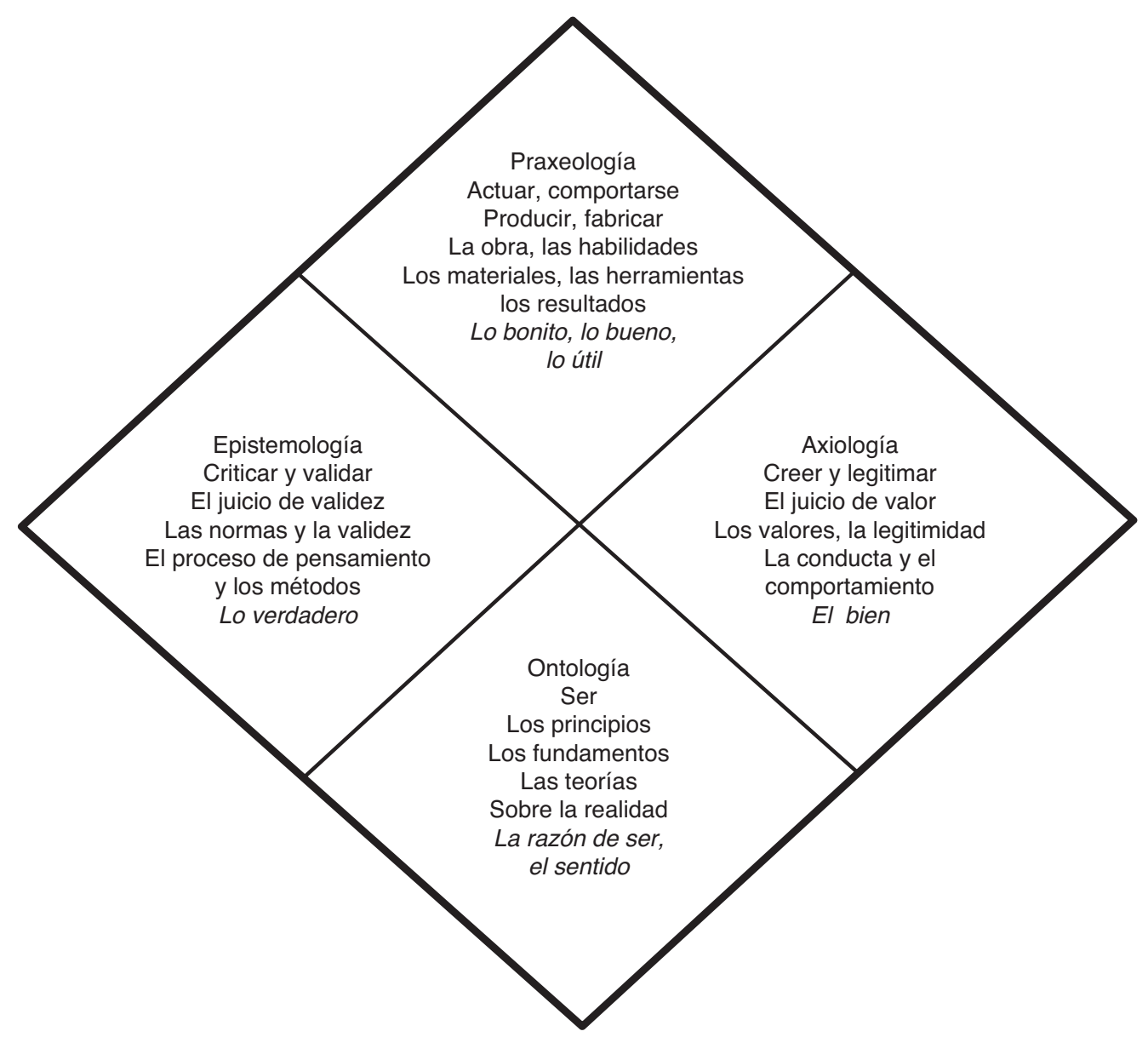

Figura 1. Los 4 fundamentos filosóficos.

Fuente: Bédard (2003) 
En las clasificaciones para propósitos de este trabajo se hace un enfoque específico en los fundamentos filosóficos para el administrador. Acogiendo a Bédard, se reconoce el lugar central del ser humano en general y se tienen en cuenta las particularidades individuales, lo cual conlleva comprender sus características, respetar y valorar los potencialidades específicas de las personas, que son esenciales para la producción del equipo (Bédard, 2003).

En el ejercicio de la administración se tienen 4 componentes: el primero de ellos el praxeológico, con el que se permite construir y validar el conocimiento en el día a día; es la etapa para crear, actuar, conducir, fabricar materiales, herramientas. Es la propia para la realización de las obras y resultados, pero esto está fundamentado en el componente epistemológico que se encarga de la filosofía del conocimiento, de las ciencias. Este permite evaluar la solidez de los conocimientos intelectuales y conceptuales sobre los que se apoyan las prácticas. La epistemología, por su parte, valida los procesos, la metodología y la teoría. A su vez, estos fundamentos deben soportarse en el elemento axiológico que es el que hace referencia a los valores morales, culturales y éticos tanto individuales como colectivos. El campo de la ética y la moral es el espacio del bien (fig. 1).

Como se puede observar, el elemento praxeológico se fundamenta en los conocimientos, en la ética y la moral pero el piso fundamental es la ontología que, como afirma Bédard (2003, p. 83): «Ella es el componente más profundo del marco de referencia, del sustrato en el que las ideas y las concepciones de la naturaleza general de la persona tienen sus raíces».

Tabla 1

Competencias genéricas

\begin{tabular}{|c|c|}
\hline Código & Descripción \\
\hline $\mathbf{1 A}$ & Compromiso ético \\
\hline $\mathbf{2 A}$ & Capacidad de aplicar los conocimientos en la práctica \\
\hline 3A & Capacidad para tomar decisiones \\
\hline $4 \mathrm{~A}$ & Compromiso con la calidad \\
\hline $\mathbf{5 A}$ & Capacidad para identificar, planear y resolver problemas \\
\hline 6A & Capacidad de abstracción, análisis y síntesis \\
\hline 7A & Conocimientos sobre el área de estudio y la profesión \\
\hline $\mathbf{8 A}$ & Capacidad de trabajo en equipo \\
\hline 9A & Capacidad de aprender y actualizarse permanentemente \\
\hline 10A & Capacidad de comunicación oral y escrita \\
\hline 11A & Responsabilidad social y compromiso ciudadano \\
\hline 12A & Capacidad para formular y gestionar proyectos \\
\hline 13A & Capacidad creativa \\
\hline $14 \mathrm{~A}$ & Capacidad para organizar y planificar el tiempo \\
\hline 15A & Habilidades en el uso de las TIC \\
\hline 16A & Capacidad de motivar y conducir hacia metas comunes \\
\hline 17A & Capacidad para actuar en nuevas situaciones \\
\hline 18A & Habilidades para buscar, procesar y analizar información procedente de fuentes diversas \\
\hline 19A & Capacidad crítica y autocrítica \\
\hline 20A & Habilidad para trabajar en contextos internacionales \\
\hline $21 G$ & Capacidad de investigación \\
\hline 22G & Habilidad para trabajar en forma autónoma \\
\hline 23G & Compromiso con la prevención del medio ambiente \\
\hline 24G & Habilidades interpersonales \\
\hline 25G & Capacidad de comunicación en un segundo idioma \\
\hline 26G & Valoración y respeto por la diversidad y multiculturalidad \\
\hline 27G & Compromiso con su medio sociocultural \\
\hline
\end{tabular}

El código de cada competencia será utilizado en las figuras para facilitar su ubicación y el análisis.

Fuente: Elaboración propia a partir de Beneitone et al. (2007). 


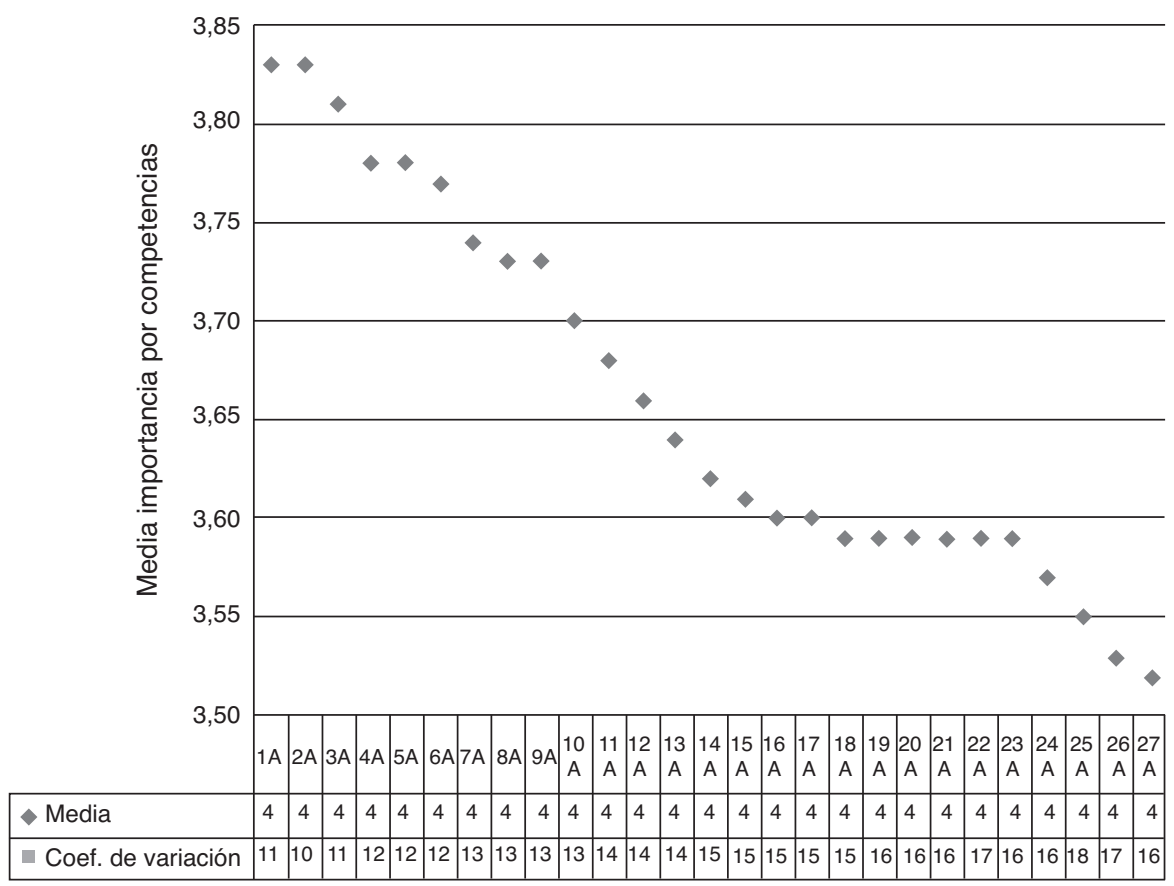

Figura 2. Ranking de importancia de las competencias genéricas: académicos Fuente: Elaboración propia.

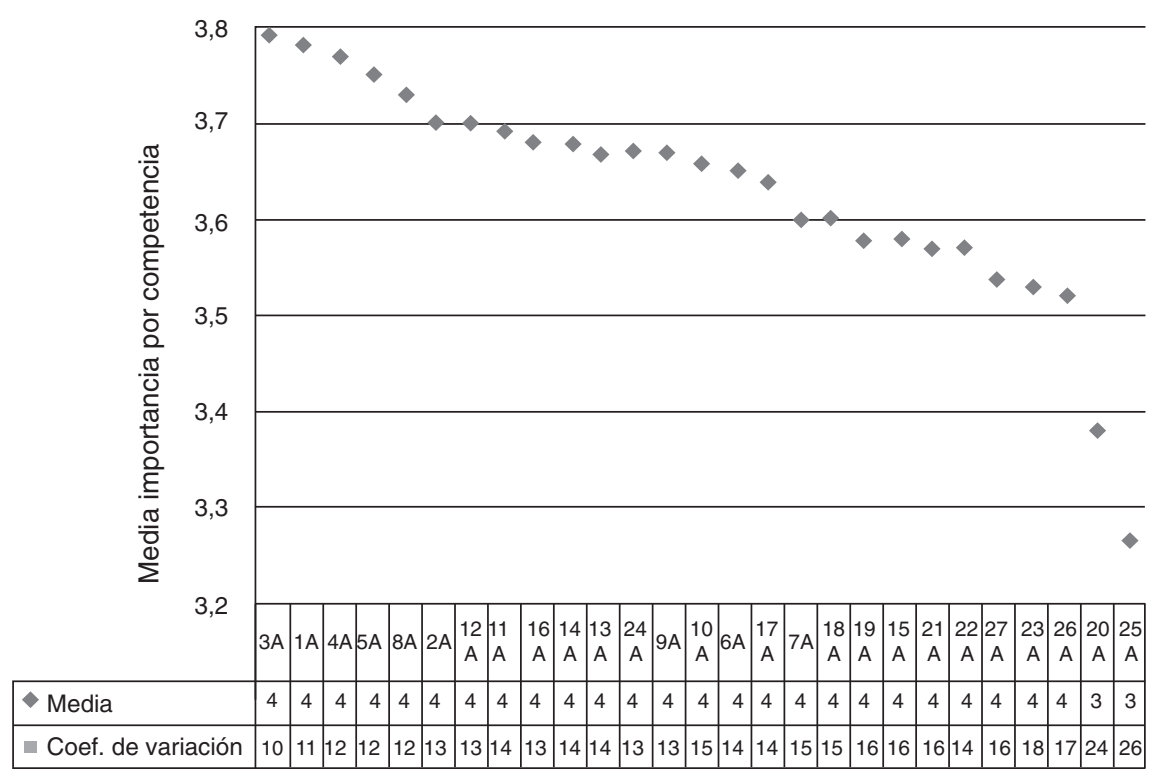

Figura 3. Ranking de importancia de las competencias genéricas: empleadores

Fuente: Elaboración propia. 
Según Bédard (2003), los 4 componentes del rombo no están separados, son aspectos puntuales de una misma realidad. Esto es que las 4 dimensiones filosóficas permiten recoger integralmente el hecho social total. La epistemología y la axiología según Bédard (2003) ejercen, cada una a su manera, una función de vigilancia crítica. La epistemología busca dar seguridad, certificar, ratificar, evaluar la solidez de los fundamentos conceptuales e intelectuales, revisa los procedimientos y métodos utilizados, permite emitir juicios sobre los conceptos y soportes en los que se apoya la práctica. La axiología valida, legitima la praxeología, vela desde el punto de vista de la conciencia del bien y el mal, de la moral, de la virtud y de las costumbres concretas en particular. Es decir, la epistemología le da un soporte a la praxis, la valida con conceptos, teorías, métodos científicos, la axiología la legítima soportándose en valores personales, sociales y culturales; sobre el bien o el mal. Pero para poder explicar la praxeología, la epistemología y la axiología hay que apoyarse en la ontología, que permite el conocimiento del ser y aborda los fundamentos conceptuales de la realidad, es la base sobre la cual cada persona construye lo conceptual, lo ético, lo crítico y lo práctico.

No importa la actividad humana, la praxeología justifica, muestra la realidad de la función administrativa. La praxis favorece la acción recíproca de las 4 dimensiones y propicia que los administradores encuentren condiciones aceptables para ejercer plenamente todas sus competencias.

El análisis de los conceptos y clasificaciones de competencias es amplio, por lo que para este trabajo se asume un concepto eclético, en línea con los postulados de Tuning y bajo el enfoque de los fundamentos filosóficos de Bédard que se identificarán en la metodología.

Tabla 2

Competencias específicas

\begin{tabular}{ll}
\hline Código & Descripción \\
\hline 1A & Desarrollar un planteamiento estratégico, táctico y operativo \\
2A & Ejercer el liderazgo para el logro y consecución de metas en la organización \\
3A & Detectar oportunidades para emprender nuevos negocios o desarrollar nuevos productos \\
4A & Administrar y desarrollar el talento humano en la organización \\
5A & Tomar decisiones de inversión, financiamiento y gestión de recursos financieros en la empresa \\
6A & Interpretar la información contable y la información financiera para la toma de decisiones gerenciales \\
7A & Elaborar, evaluar y administrar proyectos empresariales en diferentes tipos de organizaciones \\
8A & Mejorar e innovar los procesos administrativos \\
9A & Utilizar las tecnologías de información y comunicación en la gestión \\
10A & Identificar y administrar los riesgos de negocios de las organizaciones \\
11A & Usar la información de costos para el planteamiento, el control y la toma de decisiones \\
12A & Identificar y optimizar los procesos de negocios de las organizaciones \\
13A & Desarrollar, implementar y gestionar sistemas de control administrativo \\
14A & Identificar aspectos éticos y culturales de impacto recíprocos entre la organización y el entorno social \\
15A & Identificar las interrelaciones funcionales de la organización \\
16A & Formular planes de marketing \\
17A & Formular y optimizar sistemas de información para la gestión \\
18A & Administrar un sistema logístico integral \\
19A & Administrar la infraestructura tecnológica de una empresa \\
20A & Evaluar el marco jurídico aplicado a la gestión empresarial \\
\hline
\end{tabular}

El código de cada competencia será utilizado en las figuras para facilitar su ubicación y el análisis.

Fuente: Elaboración propia a partir de Beneitone et al. (2007). 
Tabla 3

Pruebas de hipótesis para académicos: competencias genéricas más importantes

\begin{tabular}{|c|c|c|c|c|c|c|c|}
\hline & \multicolumn{7}{|c|}{ Prueba para una muestra } \\
\hline & & \multicolumn{6}{|c|}{ Valor de prueba $=3$} \\
\hline & & \multirow[t]{2}{*}{$\mathrm{t}$} & \multirow[t]{2}{*}{ Gl } & \multirow[t]{2}{*}{ Sig. (bilateral) } & \multirow[t]{2}{*}{$\begin{array}{l}\text { Diferencia de } \\
\text { medias }\end{array}$} & \multicolumn{2}{|c|}{$\begin{array}{c}\text { 95\% Intervalo de confianza para } \\
\text { la diferencia }\end{array}$} \\
\hline & & & & & & Inferior & Superior \\
\hline \multirow[t]{5}{*}{ Más importantes } & Compromiso ético & 67.323 & 1,215 & 0.000 & 0.832 & 0.81 & 0.86 \\
\hline & $\begin{array}{l}\text { Capacidad de aplicar los conocimientos } \\
\text { en la práctica }\end{array}$ & 69.101 & 1,220 & 0.000 & 0.828 & 0.80 & 0.85 \\
\hline & Capacidad para tomar decisiones & 62.099 & 1,216 & 0.000 & 0.805 & 0.78 & 0.83 \\
\hline & Compromiso con la calidad & 58.481 & 1,210 & 0.000 & 0.778 & 0.75 & 0.80 \\
\hline & $\begin{array}{l}\text { Capacidad para identificar, planear y } \\
\text { resolver problemas }\end{array}$ & 59.275 & 1,220 & 0.000 & 0.780 & 0.75 & 0.81 \\
\hline
\end{tabular}

Fuente: Elaboración propia. 
Tabla 4

Pruebas de hipótesis para empleadores: competencias genéricas más importantes

\begin{tabular}{|c|c|c|c|c|c|c|c|}
\hline & \multicolumn{7}{|c|}{ Prueba para una muestra } \\
\hline & & \multicolumn{6}{|c|}{ Valor de prueba $=3$} \\
\hline & & \multirow[t]{2}{*}{$\mathrm{t}$} & \multirow[t]{2}{*}{ Gl } & \multirow[t]{2}{*}{ Sig. (bilateral) } & \multirow[t]{2}{*}{$\begin{array}{l}\text { Diferencia de } \\
\text { medias }\end{array}$} & \multicolumn{2}{|c|}{$\begin{array}{c}\text { 95\% Intervalo de confianza para } \\
\text { la diferencia }\end{array}$} \\
\hline & & & & & & Inferior & Superior \\
\hline \multirow[t]{5}{*}{ Más importantes } & Capacidad para tomar decisiones & 28.107 & 217 & 0.000 & 0.794 & 0.74 & 0.85 \\
\hline & Compromiso ético & 25.423 & 217 & 0.000 & 0.775 & 0.72 & 0.84 \\
\hline & Compromiso con la calidad & 23.946 & 214 & 0.000 & 0.772 & 0.71 & 0.84 \\
\hline & $\begin{array}{l}\text { Capacidad para identificar, planear y } \\
\text { resolver problemas }\end{array}$ & 24.494 & 217 & 0.000 & 0.752 & 0.69 & 0.81 \\
\hline & Capacidad de trabajo en equipo & 23.502 & 218 & 0.000 & 0.726 & 0.67 & 0.79 \\
\hline
\end{tabular}

Fuente: Elaboración propia. 
Tabla 5

Pruebas de hipótesis para académicos: competencias específicas más importantes

\begin{tabular}{|c|c|c|c|c|c|c|c|}
\hline & \multicolumn{7}{|c|}{ Prueba para una muestra } \\
\hline & & \multicolumn{6}{|c|}{ Valor de prueba $=3$} \\
\hline & & \multirow[t]{2}{*}{$\mathrm{t}$} & \multirow[t]{2}{*}{ Gl } & \multirow[t]{2}{*}{ Sig. (bilateral) } & \multirow[t]{2}{*}{$\begin{array}{l}\text { Diferencia de } \\
\text { medias }\end{array}$} & \multicolumn{2}{|c|}{$\begin{array}{l}\text { 95\% Intervalo de confianza para } \\
\text { la diferencia }\end{array}$} \\
\hline & & & & & & Inferior & Superior \\
\hline \multirow[t]{4}{*}{ Más importantes } & $\begin{array}{l}\text { Desarrollar un planteamiento estratégico, } \\
\text { táctico y operativo }\end{array}$ & 52.395 & 1,127 & 0.000 & 0.763 & 0.73 & 0.79 \\
\hline & $\begin{array}{l}\text { Ejercer el liderazgo para el logro y } \\
\text { consecución de metas en la organización }\end{array}$ & 48.432 & 1,133 & 0.000 & 0.737 & 0.71 & 0.77 \\
\hline & $\begin{array}{l}\text { Detectar oportunidades para emprender } \\
\text { nuevos negocios o desarrollar nuevos } \\
\text { productos }\end{array}$ & 46.780 & 1,130 & 0.000 & 0.718 & 0.69 & 0.75 \\
\hline & $\begin{array}{l}\text { Administrar y desarrollar el talento } \\
\text { humano en la organización }\end{array}$ & 46.366 & 1,132 & 0.000 & 0.715 & 0.68 & 0.75 \\
\hline
\end{tabular}

Fuente: Elaboración propia. 
Tabla 6

Pruebas de hipótesis para empleadores: competencias específicas más importantes

\begin{tabular}{|c|c|c|c|c|c|c|c|}
\hline & \multicolumn{7}{|c|}{ Prueba para una muestra } \\
\hline & & \multicolumn{6}{|c|}{ Valor de prueba $=3$} \\
\hline & & \multirow[t]{2}{*}{$\mathrm{t}$} & \multirow[t]{2}{*}{$\mathrm{Gl}$} & \multirow[t]{2}{*}{ Sig. (bilateral) } & \multirow[t]{2}{*}{$\begin{array}{l}\text { Diferencia de } \\
\text { medias }\end{array}$} & \multicolumn{2}{|c|}{$\begin{array}{c}\text { 95\% Intervalo de confianza para la } \\
\text { diferencia }\end{array}$} \\
\hline & & & & & & Inferior & Superior \\
\hline \multirow[t]{5}{*}{ Más importantes } & $\begin{array}{l}\text { Desarrollar un planteamiento estratégico, } \\
\text { táctico y operativo }\end{array}$ & 23.680 & 237 & 0.000 & 0.765 & 0.70 & 0.83 \\
\hline & $\begin{array}{l}\text { Ejercer el liderazgo para el logro y } \\
\text { consecución de metas en la organización }\end{array}$ & 26.249 & 237 & 0.000 & 0.761 & 0.70 & 0.82 \\
\hline & $\begin{array}{l}\text { Administrar y desarrollar el talento humano } \\
\text { en la organización }\end{array}$ & 21.176 & 237 & 0.000 & 0.723 & 0.66 & 0.79 \\
\hline & $\begin{array}{l}\text { Identificar y optimizar los procesos de } \\
\text { negocios de las organizaciones }\end{array}$ & 18.209 & 238 & 0.000 & 0.669 & 0.60 & 0.74 \\
\hline & $\begin{array}{l}\text { Interpretar la información contable y la } \\
\text { información financiera para la toma de } \\
\text { decisiones gerenciales }\end{array}$ & 16.935 & 238 & 0.000 & 0.669 & 0.59 & 0.75 \\
\hline
\end{tabular}




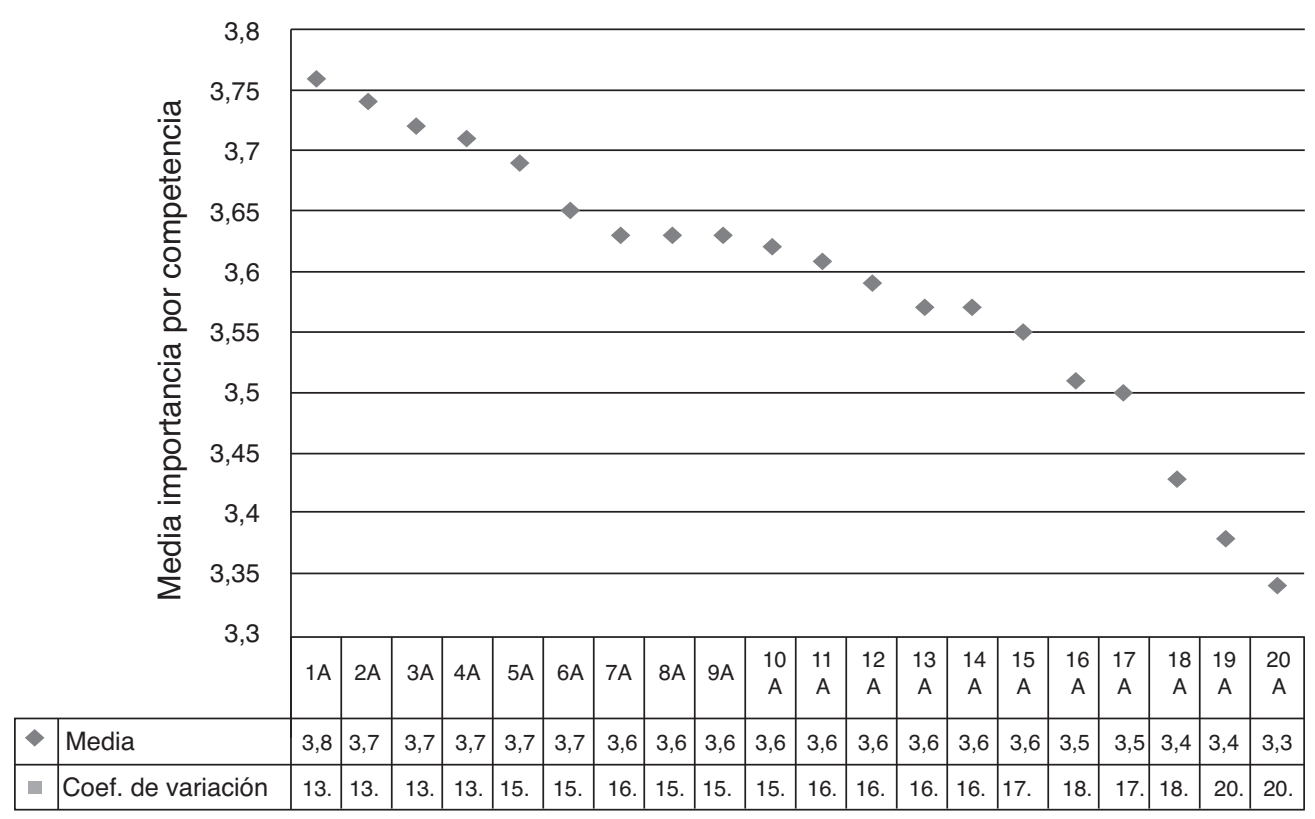

Figura 4. Ranking de importancia de las competencias específicas: académicos Fuente: Elaboración propia.

\section{Metodología}

En este trabajo se utiliza un enfoque descriptivo en la determinación y la clasificación de la importancia de las competencias; además se aplica la inferencia estadística para identificar el nivel de importancia de las competencias.

Para esta investigación se utiliza la base datos de los académicos y empleadores obtenidas en Tuning Colombia, donde se recogió su percepción, identificando la importancia y el desarrollo de 27 competencias genéricas (tabla 1) y 20 específicas (tabla 2), que son las requeridas en la formación de los estudiantes de los programas de administración. La metodología de Tuning Europa se acogió en Latinoamérica y se replicó en Colombia. Para esto se consultó a una muestra de 1.453 encuestados la importancia y desarrollo de las competencias genéricas. La muestra se distribuye de la siguiente forma: 1.234 académicos y 219 empleadores; y para las 20 competencias específicas se utilizó una muestra de 1.385 encuestados distribuida en 1.146 académicos y 239 empleadores. La escala utilizada en la investigación en Colombia para medir importancia/desarrollo de cada competencia es ordinal de 1 a 4 donde 1 representa ningún desarrollo/importancia y 4 máximo desarrollo/importancia.

Dado que las poblaciones no son normales, nos basamos en el teorema del límite central que explica que, para muestras grandes $(n>30)$, en poblaciones no normales con funciones de distribución desconocida, su distribución muestral de medias se comporta como una normal:

$$
\mathrm{N} \rightarrow\left(\bar{X} ; \frac{S}{\sqrt{n}}\right)
$$

El análisis y los resultados se presentan en 4 etapas: 1) se clasifican las competencias genéricas y específicas de acuerdo con la media ponderada de la importancia de la competencia según la 


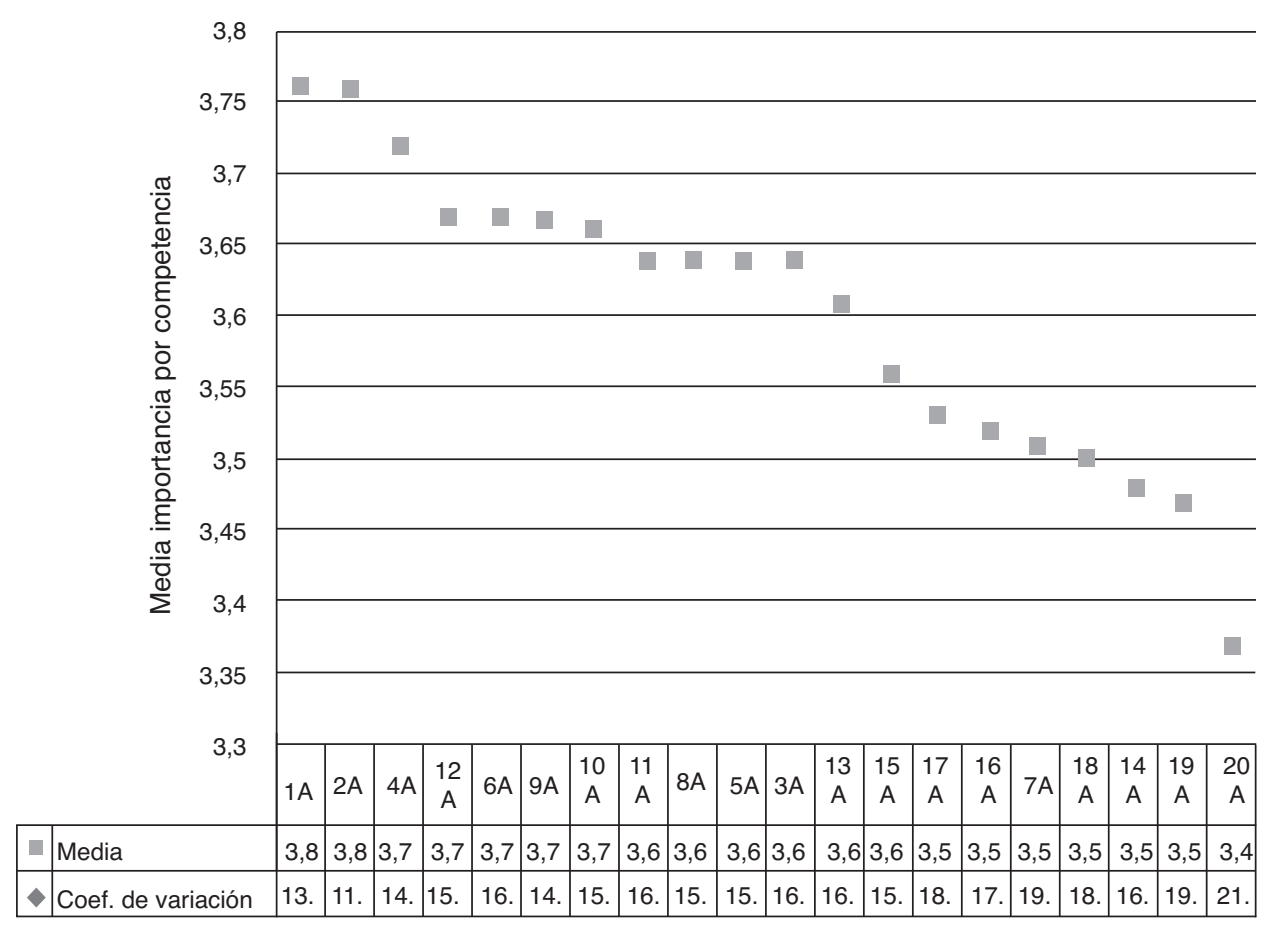

Figura 5. Ranking de importancia de las competencias específicas: empleadores Fuente: Elaboración propia.

percepción de los académicos y de los empleadores; 2) se realiza una prueba de hipótesis para comprobar el nivel de importancia asociado a las 5 competencias más importantes; 3 ) el grupo de competencias de la etapa 2 se ordena y se asocia el nivel de desarrollo de cada competencia; 4) por último, las competencias seleccionadas se clasifican de acuerdo con el enfoque de fundamentos filosóficos (Bédard, 2003).

\section{Análisis y resultados}

\section{Primera etapa}

Esta etapa tiene como objetivo determinar la clasificación de las competencias genéricas y específicas de acuerdo con la percepción de los académicos y empleadores.

Analizando la media de cada competencia genérica, vemos que los académicos muestran una puntuación superior, en otras palabras, son más optimistas. Las primeras 5 competencias muestran una media entre 3.78 y 3.83 para los académicos y entre 3.73 y 3.79 para los empleadores. De las 5 competencias con mayor promedio en importancia para los académicos (ver fig. 2), 4 están incluidas en la lista de los 5 primeras de los empleadores (véase la fig. 3).

Aunque en un orden diferente: la capacidad de aplicar los conocimientos en el campo, para los académicos fue la $2 .^{\circ}$ más importante, pero para los empresarios está en el $6 .^{\circ}$ lugar. La opinión es más homogénea en los académicos, ya que muestran menor coeficiente de variación. Una de las competencias, en desacuerdo entre las 5 primeras, es la capacidad de trabajar en equipo: para 


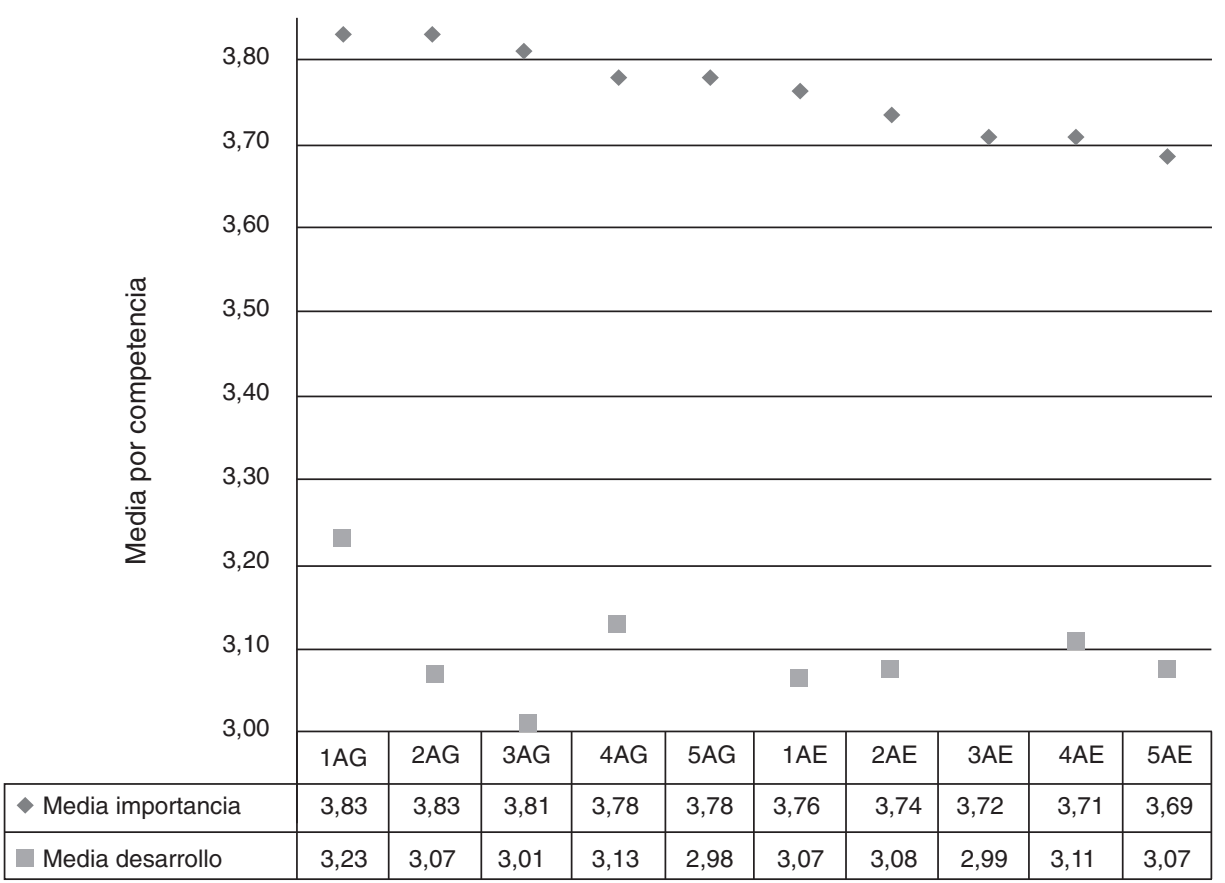

Figura 6. Competencias genéricas y específicas más importantes y su desarrollo según académicos Fuente: Elaboración propia.

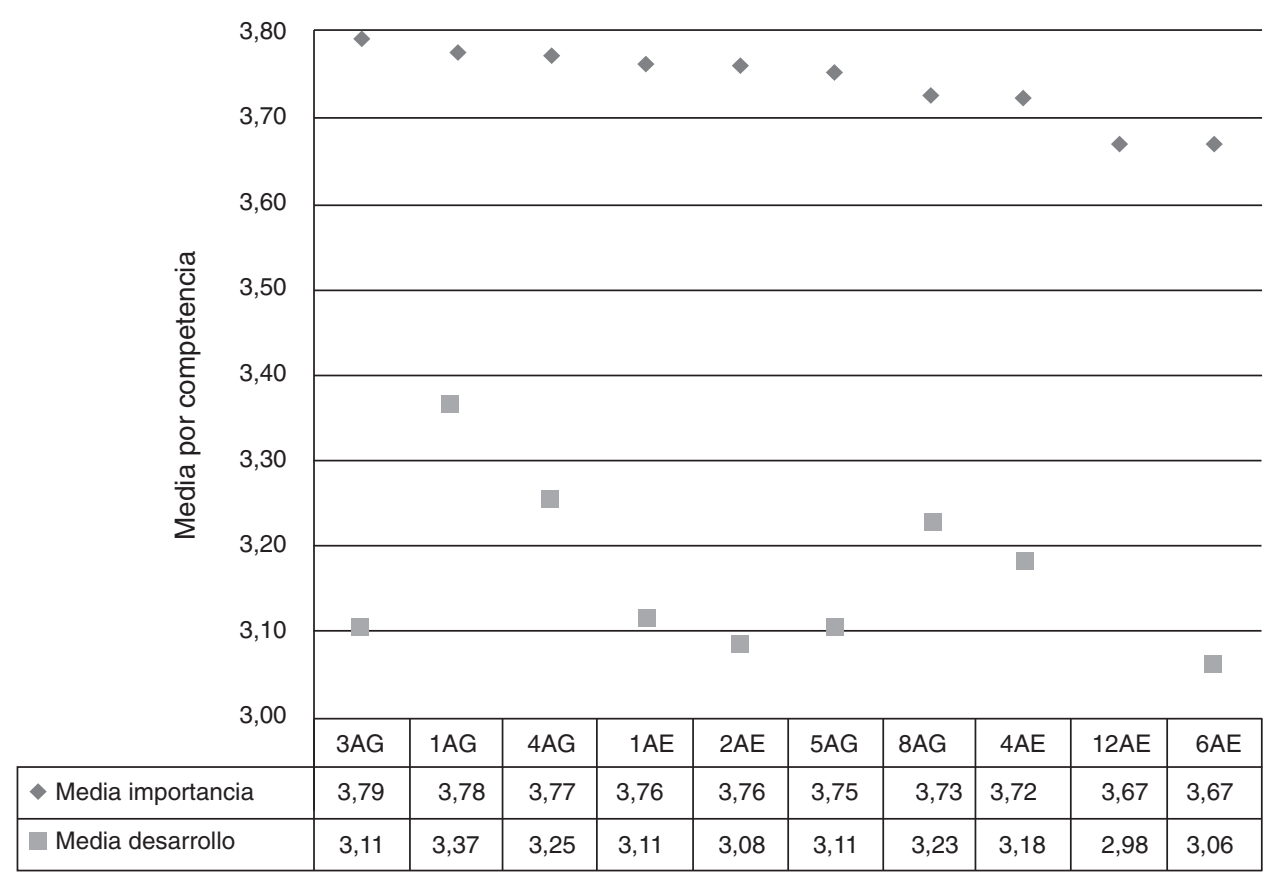

Figura 7. Competencias genéricas y específicas más importantes y su desarrollo según empleadores Fuente: Elaboración propia. 


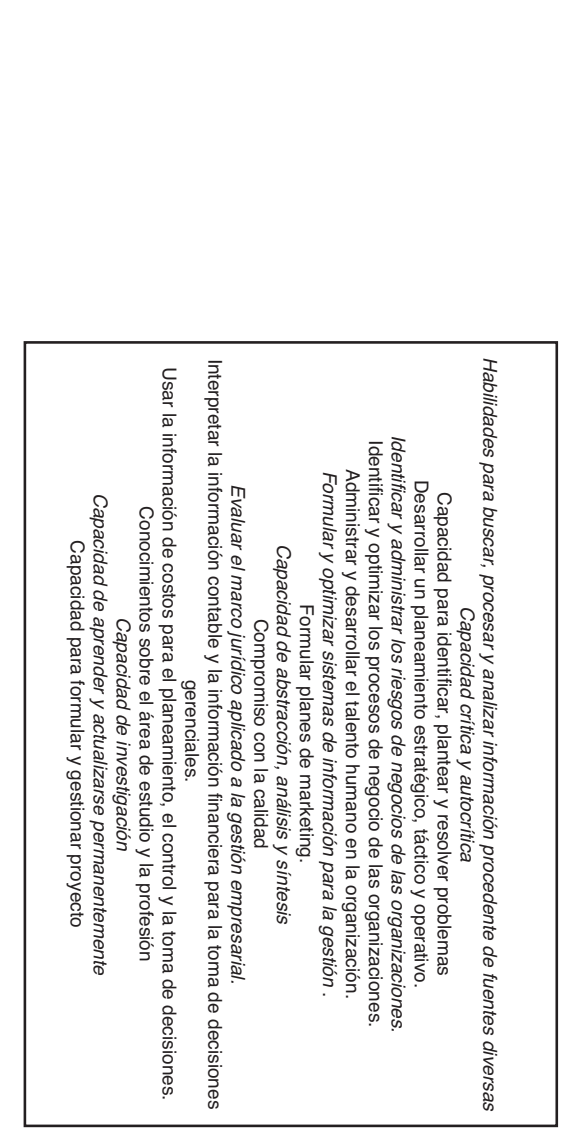

Nota: Las competencias por

desarrollar están escritas en cursiva.
Administrar un sistema logístico integral.

Desarrollar, implementar y gestionar sistemas de control administrativo.

Elaborar, evaluar y administrar proyectos empresariales en diferentes tipos de organizaciones.

Tomar decisiones de inversión, financiamiento y gestión de recursos financieros en la empresa.

Mejorar e innovar los procesos administrativos.

Administrar la infraestructura tecnológica de una empresa.

Capacidad para organizar y planificar el tiempo.

Capacidad de comunicación oral y escrita.

Capacidad para tomar decisiones

Detectar oportunidades para emprender nuevos negocios y/o desarrollar nuevos productos. Capacidad para actuar en nuevas situaciones

Habilidad para trabajar en contextos internacionales

Capacidad de aplicar los conocimientos en la práctica

Capacidad de comunicación en un segundo idioma
Habilidades en el uso de las tecnologías de la información y de la comunicación
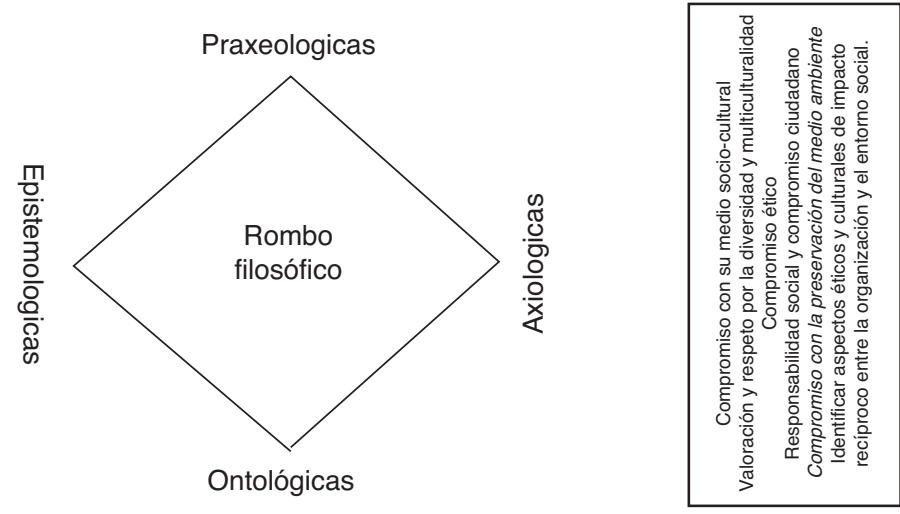

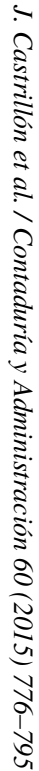

var y conducir hacia metas

Identificar las interrelaciones funcionales de la organización.

Ejercer el liderazgo para el logro y consecución de metas en la

organización.

Habilidades interpersonales

Figura 8. Competencias gerenciales de Tuning a la luz del rombo filosófico de Bédard.Fuente: Elaboración propia 


\begin{tabular}{|c|}
\hline Comun \\
\hline \hline Academicos \\
\hline \hline Empleadores \\
\hline
\end{tabular}
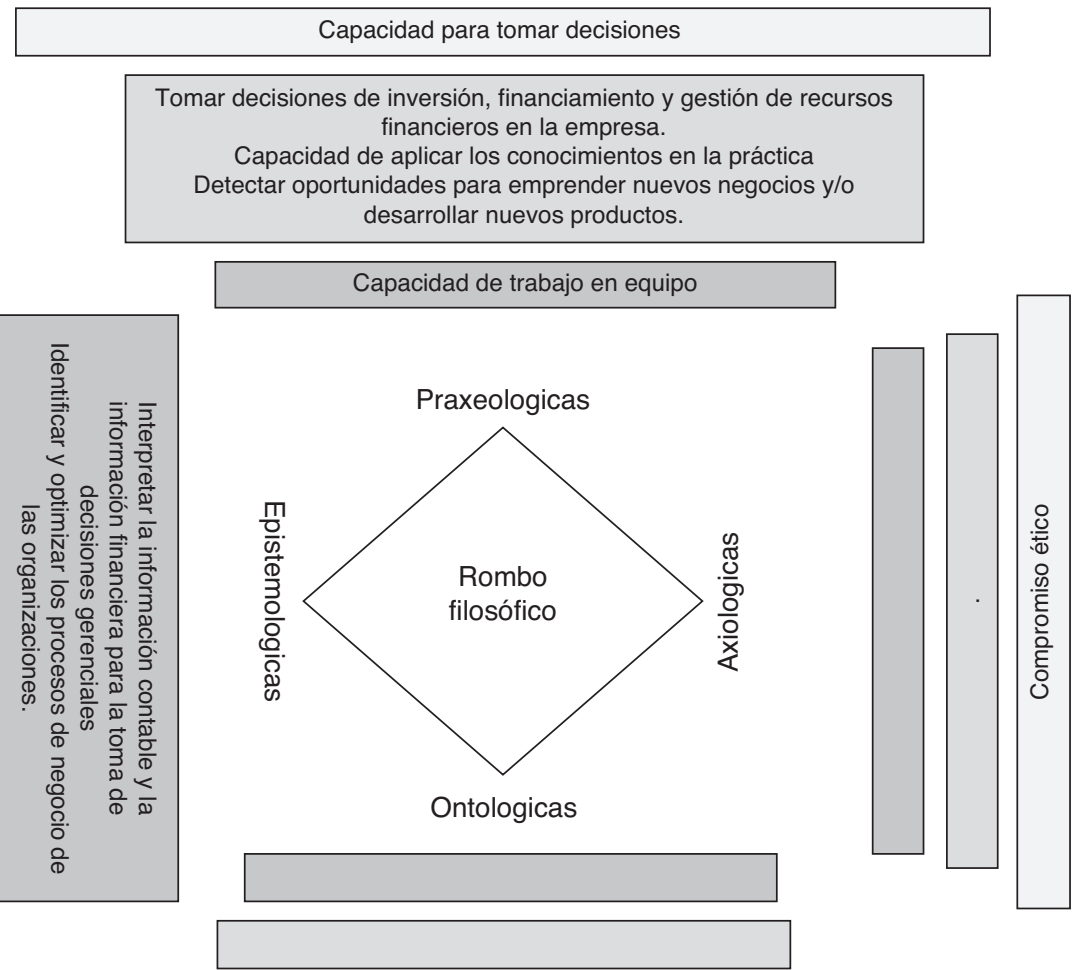

Ejercer el liderazgo para el logro y consecución de metas en la organización.

Figura 9. Competencias más importantes de acuerdo con la percepción de empleadores y académicos.

Fuente: Elaboración propia 
los empleadores es la $5 .^{\circ}$ en importancia, pero para los académicos está en el $8 .^{\circ}$ lugar, con igual homogeneidad en la percepción de los empleadores y académicos.

Entre las 5 competencias específicas más importantes para los académicos (ver fig. 4) solo 3 son comunes a los empleadores (ver fig. 5), en un orden diferente. En el 3. ${ }^{\text {er }}$ puesto con promedio de 3.72 para los académicos, detectar oportunidades para emprender nuevos negocios o desarrollar nuevos productos, se ubica según percepción de empleadores en el puesto 11 con un promedio de 3.64. Y en el 5. ${ }^{\circ}$ puesto para los académicos está tomar decisiones sobre inversiones, financiación y gestión de los recursos financieros en la organización, que se clasificó en el puesto 10 para los empleadores con promedio 3.69. Los empleadores clasificaron como 4. ${ }^{\circ}$ la competencia identificar y optimizar los procesos de los negocios de las organizaciones, pero para los académicos está en el puesto 12 .

\section{Segunda etapa}

Para las 5 competencias genéricas y específicas con la media más alta tanto para los académicos como para los empleadores se procede a comprobar la hipótesis:

Ho $=\mu \leq 3$ y Ha: $\mu>3$ bajo un nivel de significación de 0,05. Usando el SPSS, los resultados respectivos se muestran en las tablas 3-6.

Como se puede observar, las 10 competencias seleccionadas por los empleadores y académicos tienen un valor $p$ de cero, lo que indica que obtener una muestra con una media igual o inferior a 3 no es posible a cualquier nivel de significación. Luego, se rechaza la hipótesis nula y se acepta la alterna. Es decir, es significativa la afirmación de que la media de la importancia de las competencias es superior a 3. Por lo tanto, podemos concluir que estas competencias de acuerdo con la percepción de académicos y empresarios son importantes o bastante importantes para el desempeño de los administradores. Además, si miramos los límites superior e inferior de los intervalos de confianza de las competencias, vemos que son mayores que cero. Esto implica que la media de la población es mayor que 3, lo que significa que las competencias son muy importantes para los 2 grupos de análisis.

\section{Tercera etapa}

Tomando el ranking de importancia de las competencias genéricas y específicas realizado en el paso anterior, se presenta la percepción de desarrollo de los académicos y empleadores. En la figura 6 se muestra la importancia y desarrollo de las competencias específicas y genéricas seleccionadas como las más importantes para los académicos y en la figura 7 las de los empleadores. La letra $\mathrm{G}$ que acompaña el número de la competencia identifica las competencias genéricas y la letra E identifica las competencias específicas.

Con base en los resultados de Cabeza et al. (2012), que determinan el nivel de desarrollo de estas competencias, las competencias genéricas y específicas con mayor promedio están desarrolladas o bastante desarrolladas, excepto (3AE para académicos) detectar oportunidades para emprender nиevos negocios o desarrollar nuevos productos y (12AE para empleadores) identificar y optimizar los procesos de negocios de las organizaciones con un promedio de desarrollo de 2.99 y 2.98 , respectivamente.

Analizando los gráficos, se nota que los empresarios tienen diferentes órdenes de importancia de las competencias con respecto a los académicos y añaden las competencias: (8AG), capacidad de trabajo en equipo; (6AE), interpretar la información contable y la información financiera para la toma de decisiones gerenciales y (12AE), identificar y optimizar los procesos de negocios 
de las organizaciones. Estas competencias son más importantes para los empleadores que (2AG) capacidad de aplicar los conocimientos en la práctica; (3AE) detectar oportunidades para emprender nuevos negocios o desarrollar nuevos productos y (5AE) tomar decisiones de inversión, financiamiento y gestión de recursos financieros en la empresa.

\section{Cuarta etapa}

Usando el marco filosófico de Bédard, se clasifican las competencias: ontológica, axiológica, epistemológica y praxeológica (fig. 8) según Lombana, Cabeza, y Castrillón (2012).

Buscando en la figura 9 la distribución de las competencias más importantes para los académicos, el $40 \%$ son epistemológicas, el $40 \%$ praxeológicas, $10 \%$ axiológicas y $10 \%$ ontológicas. Para los empleadores, el $60 \%$ son epistemológicas, el $20 \%$ son praxeológicas, el $10 \%$ ontológicas y $10 \%$ axiológicas. Los empleadores y académicos consideran igualmente importantes las competencias del ser y los valores. El empresario considera más importantes las competencias prácticas que el conocimiento.

\section{Conclusiones}

Este trabajo identificó las 5 competencias genéricas y específicas más importantes para los empleadores y docentes que los administradores deben tener en Colombia. Las competencias genéricas comunes a ambos grupos son: compromiso ético; compromiso con la calidad; la capacidad de tomar decisiones; la capacidad para identificar, formular y resolver problemas. Estas competencias están en el rango de las más importantes, aunque en un orden de importancia diferente. Los académicos incluyen en el top 5 la capacidad de aplicar los conocimientos en el campo y los empleadores incluyen la capacidad para trabajar en equipo. Para los empleadores es más importante el trabajo en equipo que aplicar el conocimiento a la práctica. Los empleadores pueden pensar que esto lo puede desarrollar en el proceso de inducción que tiene el trabajador cuando inicia su vida laboral, en cambio la actitud del trabajador para relacionarse con el equipo de trabajo es esencial. Esta es la sexta competencia para los empresarios y la segunda para los académicos.

Entre las competencias específicas las más comunes son: desarrollar la planificación táctica, operativa y estratégica; utilizar el liderazgo para el logro de la meta de la organización; gestionar y desarrollar el talento humano en la organización, todas estas son habilidades esenciales para dirigir grupos. Los académicos agregaron 2: detectar oportunidades para emprender nuevos negocios o desarrollar nuevos productos y tomar decisiones sobre inversiones, financiación y gestión de los recursos financieros de la organización, en los puestos 3 y 5 respectivamente. Los empleadores en este grupo han añadido: identificar y optimizar los procesos de negocios de las organizaciones e interpretar la información contable y financiera para la toma de decisiones gerenciales. Para los empleadores son más importantes los procesos; los académicos las ubicaron en el puesto 12 y 6 respectivamente. Los empleadores utilizan la información contable y financiera para tomar decisiones, lo cual es de gran importancia.

Es de destacar que las competencias más importantes están desarrolladas según los académicos y los empleadores. Solo se deben fortalecer: identificar y optimizar los procesos de negocios de las organizaciones y detectar oportunidades para emprender nuevos negocios o desarrollar nuevos productos, que en académicos y empleadores respectivamente está muy cerca de 3 , es decir están casi desarrolladas. Como se ve en las figuras 6 y 7, las medias que muestran el desarrollo de las competencias están por encima de 3 (desarrolladas o bien desarrolladas). 
En las competencias más importantes según percepción de académicos y empleadores se identifica que el $80 \%$ son epistemológicas y praxiológicas en empleadores y académicos. También incluyeron competencias relacionadas con el ser humano y los valores en un $10 \%$ para cada una. Los empleadores incluyen un $60 \%$ en praxiológicas y un $20 \%$ en epistemológicas; los académicos las distribuyen más homogéneamente $40 \%$ en praxiológicas y $40 \%$ en epistemológicas. Es más importante el hacer, el aplicar el conocimiento para los empleadores; los académicos consideran que el conocimiento y su aplicación tienen la misma importancia en el desempeño del administrador.

Una de las competencias ontológicas más importantes y común a los empresarios y académicos es utilizar el liderazgo para el logro de los objetivos de la organización; la competencia axiológica común entre académicos y empleadores es el compromiso ético. Sin embargo, estas son las únicas. Por tanto, podríamos concluir que los valores y las competencias del ser humano, tomando en cuenta la percepción de los empresarios y académicos, no son tan importantes como deberían serlo para el desempeño de un administrador, ya que dan mayor importancia a la praxis y a la epistemología. Esto da cuenta de las preocupaciones que no solamente debe tener el sector empresarial sino también la academia en cuanto al énfasis que se debe dar a las competencias de valores (axiológicas) y del ser (ontológicas). Trabajos como este y futuros podrán servir de base para nutrir la discusión sobre la necesidad, pertinencia y prioridad que se le puede dar a este tipo de competencias, no solamente en la elaboración de currículos, sino en la inclusión dentro de los procesos de selección en las empresas.

Aunque esta investigación no apunta a la construcción de planes de estudio, se espera que los hallazgos abran puntos de discusión y ayuden en la formación en competencias y, de este modo, apoyen la exploración de metodologías que mejoren los resultados de la educación y la formación y, en un plazo más largo, sirvan para reducir la brecha detectada en muchos planes de estudio entre las competencias epistemológicas-praxiológicas (conocimientos, prácticas) y las axiológicas-ontológicas (actitudes, comportamiento ciudadano, valores morales, ética).

El desarrollo curricular exige incluir competencias axiológicas y competencias ontológicas, ya que estas son el apoyo para ser eficaz en el hacer y para la asimilación de los conocimientos para los administradores. Esto reflejaría una mejor actitud con el medio ambiente y la sociedad.

\section{Referencias}

Asociación Colombiana de Facultades de Administración, ASCOLFA, Grupo Interinstitucional de Investigación de Competencias en Administración, GRIICA. (2010). Las competencias de los administradores en Colombia a la luz del Proyecto Tuning América Latina 2008-2010. Jaime Castrillón y Leonor Cabeza Ed. Bogotá. ASCOLFA.

Bédard, R. (2003). Los fundamentos del pensamiento y las prácticas administrativas. AD-MINISTER, Revista de la Escuela de Administración, Universidad EAFIT, 3 junio-diciembre, 68-88.

Beneitone, P., Esqueti, C., González, J., Marty, M., Siufi, G. y Wagenaar, R. (2007). Reflexiones y perspectivas de la educación superior en América Latina. Informe final. In Proyecto Tuning-América Latina 2004-2007. Universidad de Deusto-Universidad de Groningen.

Boyatzis, R. (1982). The competent manager: A model for effective performance. Nueva York: John Wiley and Sons.

Bunk, G. (1994). La transmisión de las competencias en la formación y perfeccionamiento profesionales de la RFA. Revista Europea Formación Profesional., 1, 8-14.

Cabeza, L., Castrillón, J. y Lombana, J. (2012). Determinacion y análisis de las competencias por fortalecer para la gestión en Colombia. Revista Innovar, 22(46), 5-20.

Cabrera, K. y González, L. (2006). Currículo universitario basado en competencias. Barranquilla: Ediciones UniNorte.

Cabrera, K., Guzmán, J., Suárez J. y Rincón M. (2009). Programa de modernización curricular: la formación de pregrado en la Universidad del Norte: un reto del siglo XXI. Vicerrectoría académica, Dirección de Calidad y Proyectos Académicos de la Universidad del Norte-Barranquilla: Ediciones UniNorte. 
Ducci, M. (1997). El enfoque de competencia laboral en la perspectiva internacional. In Seminario Internacional sobre Formación Basada en Competencia Laboral: Situación actual y perspectivas, Guanajuato, México. Montevideo: Cinterfor.

Ferrández, A. (1997). El perfil profesional del los formadores. Departamento de Pedagogía Aplicada, Facultad de Ciencias de la Educación. Barcelona: Universidad Autónoma de Barcelona. Doc. policopiado.

Gallart, S. (1998). Competitividad, redes productivas y competencias laborales. Montevideo: Cinterfort, OIT.

Gilbert, T. (1978). Human competence - Engineering worthy performance. San Francisco: Pfeiffer.

Gonczi, A. (1997). Problemas asociados con la implementación de la educación basada en la competencia: de lo atomístico a lo holístico. In Formación basada en competencia laboral. Cinterfort, OIT.

Gonzalez, J., y Wagenaar, R. (Eds.). (2006). Tuning educational structures in Europe. Informe final - Proyecto piloto fase 2. Bilbao: La contribución de las universidades al proceso de Bolonia.

Hoffmann, T. (1999). The meanings of competency. Journal of European Industrial Training., 23/6, $275-286$.

Koshansky, J. (1998). El sistema de competencias. Training and Development Digest. Madrid [consultado 15 Ago 2014 ]. Disponible en: http://www.oitcinterfor.org/p\%C3\%A1gina-libro/definiciones-algunos-expertos

Le Boterf, G. (1994). De la competence. París: Les Editions d'Organization.

Lombana, J, Cabeza, L, Castrillón J. (2012). Gestión de competencias: Un debate académico sobre el Proyecto Tuning y sus fundamentos filosóficos. Ponencia presentada en la Asamblea XLVII de CLADEA: Educación general: Rompiendo fronteras [consultado 15 Ago 2014]. Disponible en: https://www.scribd.com/doc/124242469/2012-Lombana-CabezaCastrillon-Management-Competencies-Academic-Debate-Tuning-and-Philosophical-Foundations?secret_password= 1ppd43919w9f6wtvnfjc

Malpica, M. (2000). El punto de vista pedagógico. En A. Argüelles (comp.), Competencia laboral y educación basada en normas de competencia, (pp.123-139). México: Limusa, SEP, CNCCL y CONALEP.

Mertens, L. (1997). Competencia laboral: sistemas surgimiento y modelos. In Seminario Internacional sobre Formación Basada en Competencia Laboral: Situación actual y perspectivas. 1. Cinterfor: Guanajuato, México.

Mintzberg, H. (1973). The nature of managerial work. New York: Harper \& Row.

Mole, V., Dawson, S., Winstanley, D., Sherval, J. (1993). Researching managerial competencies. Paper presented at the British Academy of Management Annual Conference Milton Keynes, Crafting of Management Research, septiembre. United Kingdom.

Mulder, M. (2007). Competencia: la esencia y la utilización del concepto en la formación profesional inicial y permanente. Revista Europea de Formación Profesional, 1(40), 5-24.

Quillet (Ed.). (1976). Diccionario enciclopédico. Argentina: Buenos Aires.

Ruiz, M. (2005). Enfoque metodológico para la formación de competencias desde el ámbito educativo: cómo concretar una alternativa para la relevancia educativa. Universidad Distrital Francisco José de Caldas. Revista Enunciación, 10, 85-93.

Tejada, J. (1999). Acerca de las competencias profesionales (versiones I y II). Revista Herramientas, (I) $568-514$ y (II) $57,20-30$.

UNESCO. (2011) Enfoque por competencias. Oficina Internacional de Educación. [consultado 11 Jun 2014]. Disponible en: http://www.ibe.unesco.org/es/comunidades/comunidad-de-practica-cop/enfoque-por-competencias.html

Victorino L., Medina G. (2008). Educación basada en competencias y el proyecto de sintonización en Europa y Latinoamérica.Ide@s CONCYTEG. Año 3,núm. 39, 97-114.

Vygotsky, L. (1979). El desarrollo de los procesos psicológicos superiores. Barcelona: Crítica. 